\title{
Intravascular lithotripsy for heavily calcified subtotal occlusion of right coronary artery
}

\author{
Maksymilian P. Opolski ${ }^{1}$, Rafal Wolny ${ }^{1}$, Kajetan Grodecki ${ }^{1,2}$, \\ Artur Debski ${ }^{1}$, Adam Witkowski ${ }^{1}$ \\ ${ }^{1}$ Department of Interventional Cardiology and Angiology, \\ Institute of Cardiology, Warsaw, Poland \\ ${ }^{2}$ Medical University of Warsaw, Poland
}

A 64-year-old symptomatic man with $\mathrm{Ca}$ nadian Cardiovascular Society (CCS) class III angina was referred for percutaneous coronary intervention of subtotal occlusion of the ostial right coronary artery (RCA; Fig. 1A). Following intubation with a $7 \mathrm{~F} \mathrm{AL} 0.75$ guiding catheter, and sequential high-pressure predilatation $(1.2 \mathrm{~mm}$ semi-compliant balloon, and $2.0 \mathrm{~mm}$ to $3.0 \mathrm{~mm}$ non-compliant balloons), intravascular ultrasound (IVUS) revealed extensive three-to-four-quadrant $\left(270^{\circ}\right.$ to $\left.360^{\circ}\right)$ calcification within proximal RCA along with persistent ostial stenosis of the vessel (Fig. 1D-F). To modify plaque within proximal RCA, a $4.0 \times 12 \mathrm{~mm}$ intravascular lithotripsy (IVL) balloon was inflated to $4 \mathrm{~atm}$, and 8 cycles of 10 pulses each were delivered, followed by further dilatation to nominal pressure (Fig. 1B). IVUS after IVL confirmed multiple calcium disruptions (Fig. 1D'-F') allowing for guideliner-facilitated delivery and deployment of 2 drug eluting stents ( $4.0 \mathrm{~mm}$ each), and further high-pressure postdilatation (at $22 \mathrm{~atm}$ ) using a 4.5 non-compliant balloon. Optimal angiographic result (Fig. 1C) was subsequently verified with both IVUS (Fig. 1D"-F"; Suppl. Video 1) and instantaneous wave-free ratio.

Coronary IVL is a novel catheter-based technique that utilizes sonic pressure waves to disrupt calcified lesions. Herein we present a case of IVL for treatment of subtotal ostial coronary occlusion with severe calcification resulting in successful delivery and optimal expansion of coronary stents. Whether IVL may supplement available percutaneous techniques in coronary total occlusions is to be elucidated in future trials.

Conflict of interest: None declared

Address for correspondence: Dr. Maksymilian P. Opolski, Department of Interventional Cardiology and Angiology, Institute of Cardiology, ul. Alpejska 42, 04-628 Warsaw, Poland, tel: +48 501444303, fax: +48 2261338 19, e-mail: opolski.mp@gmail.com

Received: 4.06.2019 Accepted: 23.06.2019 


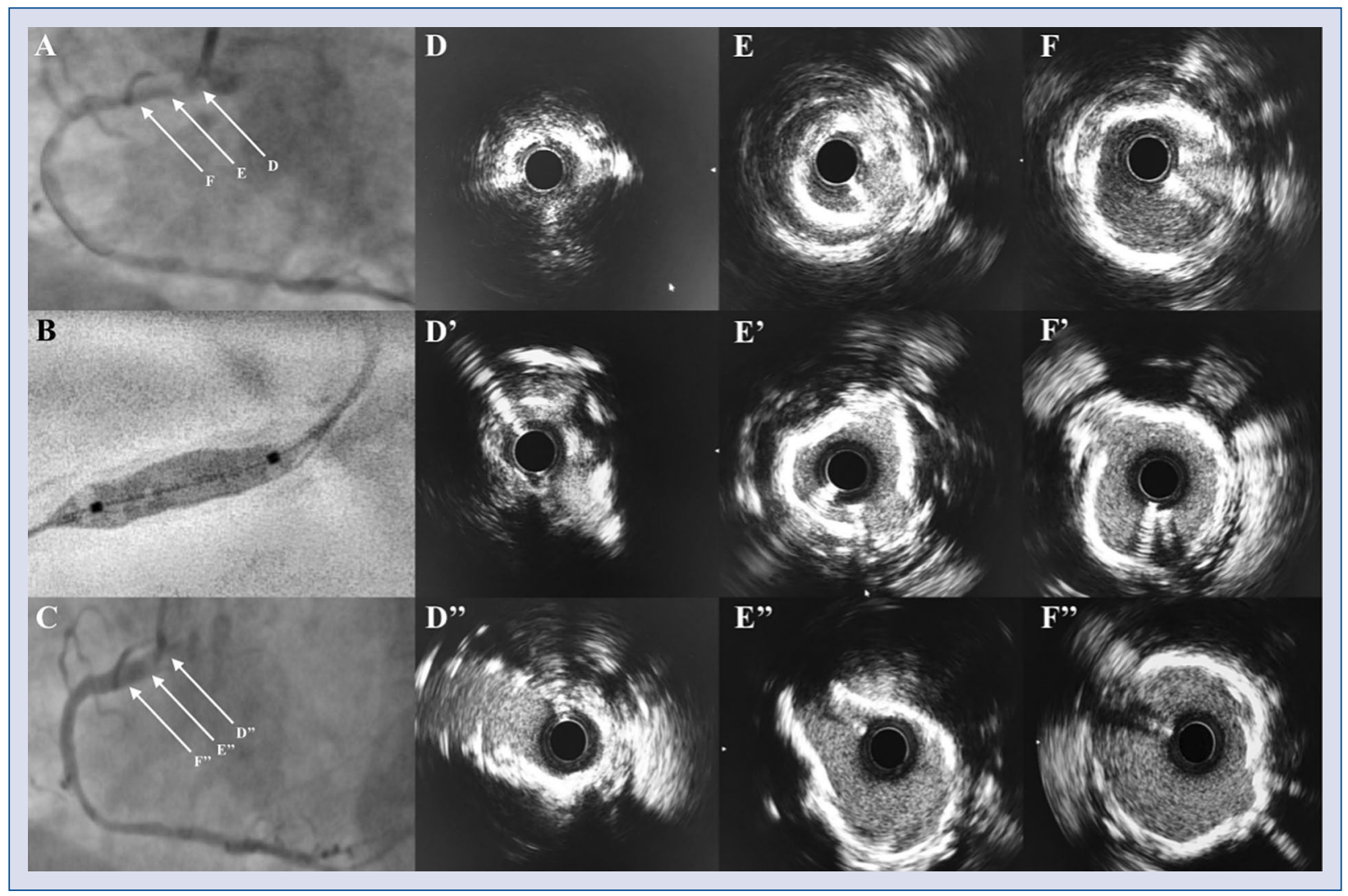

Figure 1. A. Subtotal occlusion of the ostial right coronary artery (RCA) with tortuous uptake from the aorta; B. Angiographic appearance of the Shockwave intravascular lithotripsy (IVL) balloon; C. Final angiographic result after stent implantation; D-F. Intravascular ultrasound (IVUS) before IVL revealing three-to-four-quadrant $\left(270^{\circ}\right.$ to $\left.360^{\circ}\right)$ calcification of the proximal RCA along with severe ostial stenosis; $\mathbf{D}^{\prime}-\mathbf{F}^{\prime}$. IVUS after IVL demonstrating successful fracture of the calcified lesion within proximal RCA; D"-F". Final IVUS showing optimal stent expansion. 